\title{
Heterosis and Combining Ability Analysis for Yield and Yield Attributes in Indian Mustard (Brassica juncea L.)
}

\author{
V. V. Singh*, Balbeer, H. S. Meena, Swarnim Kulshrestha, Monika Dubey, \\ Neeraj Gurjar, Pankaj Garg, M. L. Meena and P. K. Rai \\ ICAR-Directorate of Rapeseed-Mustard Research, Sewar, Bharatpur-321 303, \\ Rajasthan, India \\ *Corresponding author
}

K e y w o r d s
Brassica juncea,
GCA,
Heterobeltiosis,
Indian mustard,
Better-parent
heterosis, SCA
Article Info
Accepted:
$\begin{aligned} & 12 \text { February } 2020 \\ & \text { Available Online: } \\ & 10 \text { March } 2020\end{aligned}$

\section{A B S T R A C T}

Half diallel analysis of ten parents was performed to know the high heterotic crosses and their relationship in terms of general and specific combining ability (GCA \& SCA) in Indian mustard. The relative heterosis and heterobeltiosis were observed to be the highest with respect to siliquae on main shoot in crosses BPR-549-9 $\times$ UP-II-73 and Urvashi $\times$ NRCHB101, siliquae length in crosses UP-II-73 $\times$ NRCHB-101, UP-II-73 $\times$ Rohini and NRCHB-101 $\times$ Rohini, main shoot length in cross UP-II-73 $\times$ NRCHB-101, fruiting zone length in cross NRCHB-101 $\times$ Rohini, primary branches per plant in case of cross BPR-543-2 $\times$ Urvashi and secondary branches per plant in case of cross BPR-549-9 $\times$ EC-511664.GCA and SCA variances were significant in most of the characters. The variance of GCA $\left(\sigma^{2} \mathrm{~g}\right)$ was observed to be higher for siliquae per plant, fruiting zone length and main shoot length whereas the variance of SCA $\left(\sigma^{2} s\right)$ was higher for main shoot length and other remaining characters.

\section{Introduction}

Indian mustard $(2 \mathrm{n}=4 \mathrm{x}=36)$ is an important rabi season oilseed crop in India and occupies a premier position among the oilseed crops due to its high oil content (37-42\%). It is derived from interspecific hybridization between Brassica rapa $(2 \mathrm{n}=20)$ and Brassica nigra $(2 \mathrm{n}=16)$ followed by natural chromosome doubling. High yield and high oil content are the breeding objectives in case of mustard. There is compelling necessity to push forward and stabilize the productivity of Indian mustard.

This can be achieved through exploitation of germplasm resources and integration of genomic tools to impart efficiency and pace of breeding processes (Banga, 2012). Various breeding approaches are used for 
improvement of Brassica crops. Heterosis breeding is one of the successful breeding options being employed for the improvement of crop. Study of heterosis provides information about gene action and helps in identifying desirable gene action. Combining ability analysis involved in the inheritance of quantitative characters and in the phenomenon of heterosis is necessary for the evaluation of various possible breeding procedures (Allard,1960).

Information on combining ability helps in partitioning the total genetic variation into general combining ability of parents and specific combining ability of crosses, which is useful to assess the nature of gene action controlling different characters and devising suitable breeding strategy for improvement of the character. With this background, the present investigation was undertaken to study combining ability and heterosis of parents and their specific crosses in Indian mustard.

\section{Materials and Methods}

The experimental material comprised of ten parents viz; BPR 543-2, Urvashi, BPR 549-9, DRMR 1165-40, UP-II-73, EC 511664, NRCDR-02, NRCHB-101, Rohini and DRMR IJ-31 and their 45 half diallel crosses. The seeds of $45 \mathrm{~F}_{1}$ hybrids and ten parents were produced by hand emasculation-hand pollination and selfing, during Rabi 2016-17.

These $45 \mathrm{~F}_{1}$ hybrids along with 10 parents were evaluated in randomized block design with three replications during rabi 2017-18 at ICAR-Directorate of Rapeseed Mustard Research, Sewar, Bharatpur. Inter and intra row spacing was kept at 30 and $10 \mathrm{~cm}$, respectively. All the recommended package of practices was adopted to grow a good crop. Observations were recorded for various characters viz., plant height $(\mathrm{cm})$, number of primary branches per plant, number of secondary branches per plant, fruiting zone length $(\mathrm{cm})$, main shoot length $(\mathrm{cm})$, number of siliquae on main shoot, siliquae length (cm), number of seeds per siliquae, total siliquae per plant, oil content (\%) and seed yield per plant $(\mathrm{g})$ on five randomly selected emulative plants in every genotype in each replication. Data were subjected to diallel analysis according to Model-I, Method-II proposed by Griffing (1956).

$$
X_{i j}=u+g_{i}+g_{j}+s_{i j}+(1 / b) \sum_{k} e_{i j k},
$$

$(i=j=1 \ldots p ; k=1 \ldots b)$, where, $u$ is the population mean; $g_{i}$ is the general combining ability effect of the $i^{\text {th }}$ parent; $g_{j}$ is the general combining ability effect of the $j^{\text {th }}$ parent; $S_{i j}$ is the specific combining ability effect of the cross between $i^{\text {th }}$ and $j^{\text {th }}$ parents; $e_{i j k}$ is the environmental effect associated with $i j k^{\text {th }}$ observation.

Analysis of variance suggested by Panse and Sukhatme (1967) was followed to test the significant differences between the genotypes for all the characters. Heterosis expressed as percent increase or decrease in hybrid $\left(F_{1}\right)$ over its mid parent value and better parent value in the desirable direction was estimated for various traits as per the formula $\mathrm{RH}=100$ $\times\left[\left(\mathrm{F}_{1}-\mathrm{MP}\right) / \mathrm{MP}\right]$ suggested by Briggle (1963), $\mathrm{BPH}=100 \times\left[\left(\mathrm{F}_{1}-\mathrm{BP}\right) / \mathrm{BP}\right]$ suggested by Fonseca and Patterson (1968) respectively. Where $F_{1}=$ mean hybrid performance, $\mathrm{BP}=$ mean performance of better parents and $\mathrm{MP}=$ mean performance of mid parent.

\section{Results and Discussion}

\section{Combining ability analysis}

The analysis of variance for combining ability manifest the significance of mean squares due to gca and sca for all the traits, except gca mean square for number of primary branches 
per plant, seeds per siliquae, total siliquae per plant, oil content, and sca mean square for number of primary branches per plant and total siliquae per plant.

This indicated that both additive and nonadditive gene actions played vital role in the inheritance of these traits; whereas for seeds per siliquae and oil content, only sca mean square was observed significant, indicating the importance of non-additive gene action for the expression of these traits.

The sca variance component was observed to be higher than the respective gca variance component $\left(\sigma^{2}\right.$ gca $/ \sigma^{2}$ sca ratio $\left.<1\right)$ for all the traits, indicating the preponderance of nonadditive gene action for the inheritance of all the traits (Table 1). Similar results were also reported by Sheikh and Singh (1998), Mahto and Haider (2001), Singh et al., (2003), Gupta et al., (2011), and Meena et al., (2015).

In mustard, reduced plant height and length of main shoot are desirable traits hence; higher the negative values of GCA and SCA, better are the genotypes for breeding. In our study, maximum negative GCA value was exhibited by the genotype NRCHB 101 for plant height (-4.524) and positive GCA values for percent oil content (0.216).

Similar results are found by Teklewold, et al., (2005). Further, the genotype Rohini exhibited positive GCA for siliquae per plant (23.028), plant height (5.024), fruiting zone length (2.385) and seed per siliquae (0.258); EC511664 for number of secondary branches per plant (0.934); BPR 549-9 for main shoot length (2.754); DRMR 1165-40 for number of siliquae on main shoot (1.808) and BPR 5432 for siliquae length (0.156); DRMR-IJ-31 for fruiting zone length (-3.786), main shoot length (-2.985); UP-II-73 for number siliquae on main shoot (-2.934) and siliquae length ($0.208)$ and BPR 543-2 (-0.238) for percent oil content (Table 2). Simlarly, maximum negative SCA effect was exhibited by UP-II$73 \times$ EC-511664 (-19.08) for plant height, BPR 543-2 $\times$ Urvashi (-9.87) for main shoot length.

The highest positive SCA values were observed in cross combination of BPR 543-2 $\times$ Urvashi (1.15) for number of primary branches per plant, BPR- 549-9 × EC-511664 (5.69) for number of secondary branches per plant, BPR-549-9 × UP-II-73 (8.52) for number of siliquae on main shoot, DRMR 1165-40 $\times$ NRCDR 02 (9.63) for fruiting zone length, NRCHB $101 \times$ Rohini (0.51) for siliquae length, BPR-549-9 $\times$ NRCHB 101(0.93) for number of seeds per siliquae, BPR 543-2 × DRMR 1165-40 (95.56) for number of siliquae per plant and BPR-549-9 $\times$ NRCDR 02 (1.22) for percent oil content (Table 3).

\section{Estimation of relative heterosis and heterobeltiosis}

The estimates of heterosis calculated as percent increase or decrease over better and mid-parental values for all the studied characters in half diallel analysis are presented in Table 4.

The results revealed that, of the 45 crosses, seventeen genotypes showed positive and twenty eight genotypes showed negative heterobeltiosis for plant height with the highest value to be observed in UP-II-73 $\mathrm{x}$ EC-511664 (-18.20\%), while eighteen genotypes displayed negative relative heterosis of which UP-II-73 x EC-511664 showed the maximum $(-14.91 \%)$ relative heterosis.

These results are adorned with findings of Khulbe et al., (1998), Verma et al., (2000) and Gupta et al., (2011). 
Table.1 Analysis of variance for combing ability, estimates of components of variance and their ratio for various characters in Indian mustard

\begin{tabular}{|c|c|c|c|c|c|c|c|c|c|c|c|c|}
\hline Source & d.f. & PH & PB & SB & FZL & MSL & SOMS & SL & $\mathbf{S} / \mathbf{S}$ & $\mathbf{S} / \mathbf{P}$ & O.C. & $\mathbf{Y} / \mathbf{P}$ \\
\hline GCA & 9 & $99.511 *$ & 0.203 & $3.141^{*}$ & $56.377 * * *$ & $37.008 * *$ & $27.596 * * *$ & $0.130^{*}$ & 0.279 & 2183.594 & 0.250 & 19.853 \\
\hline error & 108 & 44.608 & 0.216 & 1.551 & 13.090 & 14.274 & 6.403 & 0.054 & 0.212 & 1395.807 & 0.149 & 12.965 \\
\hline$\sigma^{2}$ sca & & 28.958 & -0.040 & 1.238 & 10.555 & 18.085 & 6.091 & 0.074 & 0.233 & -36.139 & 0.152 & 0.824 \\
\hline $\begin{array}{c}\sigma^{2} \text { gca } / \\
\sigma^{2} \text { sca }\end{array}$ & & 0.158 & 0.026 & 0.107 & 0.342 & 0.105 & 0.290 & 0.086 & 0.024 & -1.817 & 0.055 & 0.697 \\
\hline
\end{tabular}

*Significant at $5 \%$ and $* * 1 \%$ levels respectively. Where, PH-Plant height $(\mathrm{cm})$, PB-Number of Primary branches per plant, SB- Number of secondary branches per plant, FZL- Fruiting zone length,MSL- Main shoot length, SOMS- Number of Siliquae on main shoot, SL- Siliquae length, S/S- Seeds per siliquae, S/P- Total Siliquae per plant, O.C.- Oil content, Y/P- Yield per plant.

Table.2 Estimates of gcaeffects of parental lines for 11 character in $10 X 10$ half Diallel set of Brassica juncea (L.) Czern and Coss

\begin{tabular}{|c|c|c|c|c|c|c|c|c|c|c|c|c|}
\hline S. $\mathbf{N}$. & Genotypes & PH & PB & SB & FZL & MSL & SOMS & SL & $\mathbf{S} / \mathbf{S}$ & $\mathbf{S} / \mathbf{P}$ & O.C. & $\mathbf{Y} / \mathbf{P}$ \\
\hline 1 & BPR 543-2 & 3.141 & 0.110 & 0.594 & 1.302 & -0.790 & $-1.376^{*}$ & $0.156^{*}$ & 0.011 & 7.739 & 0.564 & 1.264 \\
\hline 2 & Urvashi & -2.431 & -0.085 & 0.077 & -0.726 & -0.446 & 0.008 & -0.068 & -0.156 & 10.905 & 0.847 & -0.731 \\
\hline 3 & BPR 549-9 & 1.624 & -0.040 & 0.277 & $2.163 *$ & $2.754 * *$ & 1.269 & 0.037 & -0.036 & -1.089 & 0.889 & 1.125 \\
\hline 4 & $\begin{array}{l}\text { DRMR 1165- } \\
40\end{array}$ & 0.819 & -0.024 & -0.462 & 1.113 & 0.826 & $1.808^{*}$ & 0.088 & -0.064 & -2.017 & -0.583 & -1.592 \\
\hline 5 & UP-II-73 & -0.674 & 0.033 & -0.029 & -1.202 & -1.360 & $-2.934 * * *$ & $-0.208 * *$ & -0.127 & -15.242 & 0.864 & -0.581 \\
\hline 6 & EC 511664 & -1.661 & 0.132 & $0.934 * *$ & 0.881 & 1.732 & 1.352 & -0.025 & -0.011 & 13.936 & $-2.122 *$ & 1.525 \\
\hline 7 & NRCDR 02 & 1.038 & 0.083 & $-0.681^{*}$ & 1.131 & 1.167 & 0.708 & -0.010 & 0.136 & -14.679 & -0.731 & -0.753 \\
\hline 8 & NRCHB 101 & $-4.524 *$ & -0.196 & -0.465 & $-3.259 * *$ & -1.703 & 0.527 & -0.051 & -0.203 & -7.654 & 1.100 & -0.300 \\
\hline 9 & Rohini & $5.024 * *$ & 0.176 & 0.127 & $2.385^{*}$ & 0.804 & 0.263 & 0.109 & $0.258^{*}$ & $23.028 *$ & 1.000 & 1.700 \\
\hline \multirow[t]{7}{*}{10} & DRMR IJ 31 & -2.355 & -0.190 & -0.372 & $-3.786 * * *$ & $-2.985 * *$ & $-1.626^{*}$ & -0.027 & 0.191 & -14.925 & -1.828 & -1.658 \\
\hline & $\mathrm{SE}$ (gi)+ & 1.83 & 0.13 & 0.34 & 0.99 & 1.03 & 0.69 & 0.06 & 0.13 & 10.23 & 1.06 & 0.99 \\
\hline & CD 5\% & 4.137 & 0.29 & 0.77 & 2.24 & 2.34 & 1.57 & 0.14 & 0.29 & 23.15 & 2.41 & 2.23 \\
\hline & $\mathrm{CD} 1 \%$ & 5.94 & 0.41 & 1.11 & 3.22 & 3.36 & 2.25 & 0.21 & 0.41 & 33.25 & 3.46 & 3.20 \\
\hline & SE (gi-gj)+ & 2.73 & 0.19 & 0.51 & 1.48 & 1.54 & 1.03 & 0.09 & 0.19 & 15.25 & 1.59 & 1.47 \\
\hline & CD 5\% & 6.17 & 0.43 & 1.15 & 3.34 & 3.49 & 2.34 & 0.21 & 0.43 & 34.50 & 3.59 & 3.33 \\
\hline & $\mathrm{CD} 1 \%$ & 8.86 & 0.62 & 1.65 & 4.80 & 5.01 & 3.36 & 0.31 & 0.61 & 49.57 & 5.15 & 4.78 \\
\hline
\end{tabular}


Further, for number of primary branches per plant thirty genotypes showed positive heterobeltiosis (highest $25.00 \%$ in BPR 543-2 $\times$ Urvashi) and thirty five genotypes showed positive relative heterosis (highest $29.63 \%$ in BPR 543-2 × Urvashi).

Nineteen genotypes were found to have positive better parent heterosis for number of secondary branches per plant (highest $48.02 \%$ in BPR-549-9 × EC-511664), whereas twenty eight genotypes were found to be associated with positive mid-parent heterosis with the highest value of $72.94 \%$ in BPR-549-9 $\times$ EC511664.

The findings for number of primary branches per plant and number of secondary branches per plant are further corroborated with the results of Gupta et al., (2011). Twenty one genotypes had positive heterobeltiosis for fruiting zone length (highest 19.09 in the cross NRCHB $101 \times$ Rohini) whereas eleven crosses had negative mid-parent heterosis with the highest value of $-11.29 \%$ in DRMR1165-40 × EC-511664.

Correspondingly, in case of length of main shoots, positive better parent heterosis were shown by fifteen crosses (highest $28.57 \%$ in UP-II-73 $\times$ NRCHB 101) and mid-parent heterosis was shown by thirty crosses (highest being $29.81 \%$ in UP-II-73 $\times$ NRCHB 101); for number of siliquae on main shoot, sixteen crosses displayed positive better parent heterosis (highest 18.34\% in BPR-549-9 $\times$ UP-II-73) and twenty seven crosses exhibited positive mid-parent heterosis and highest $(30.18 \%)$ in BPR-549-9 × UP-II-73.

These results are higher than the observation of Mahto, et al., (2004) but lower than that of Mahmood et al., (2003) but confirms with the findings of Gupta et al., (2011). Moreover, twenty genotypes (highest $25.82 \%$ in NRCHB $101 \times$ Rohini) exhibited positive heterobeltiosis for siliquae length and thirty two crosses exhibited positive mid-parent heterosis and highest (32.19\%) in UP-II-73 $\times$ Rohini; Further, thirty seven out of 45 (highest $-18.06 \%$ ) and twenty four out of 45 genotypes (highest $-17.52 \%$ ) in BPR-549-9 $\times$ EC-511664 were found to have negative better and mid-parent heterosis respectively, for number of seeds per siliquae. Moreover, in case of total siliquae per plant highest of $26.62 \%$ heterobeltiosis was observed in BPR 543-2 × Urvashi amongst eleven positive crosses found and maximum of $33.36 \%$ in BPR 543-2 $\times$ Urvashi relative heterosis was recorded among the nineteen positive crosses observed.

For the trait oil content (\%) maximum heterobeltiosis was found to be $3.37 \%$ (BPR 543-2 $\times$ UP-II-73) and relative heterosis was observed as $3.42 \%$ (BPR 543-2 × UP-II-73) out of the sixteen and thirty genotypes observed to have positive better and midparent heterosis respectively.

Similar results are found by Singh et al., (2008) and Meena et al., (2014) for oil contents, seed yield and its contributing characters in Indian mustard.

For yield per plant fourteen crosses displayed positive heterobeltiosis (maximum $57.20 \%$ in BPR-543-2 × UP-II-73) and twenty two were found to possess positive relative heterosis with maximum heterosis of $61.95 \%$ in cross combination of BPR-543-2 $\times$ UP-II-73. These results are corroborated with the findings of Singh et al., (2008), Patel et al., (2012) and Meena et al., (2014).

The study indicates that these F1 hybrids could be further evaluated to obtain desirable segregants for development of superior genotypes for seed yield and its component traits through bi-parental mating or recurrent selection breeding approaches. 
Table.3 Estimates of scaeffects of parental lines for 11 character in $10 \mathrm{X} 10$ half Diallel set of Brassica juncea (L.) Czern and Coss

\begin{tabular}{|c|c|c|c|c|c|c|c|c|c|c|c|}
\hline Crosses & PH & PB & SB & FZL & MSL & SOMS & SL & $\mathbf{S} / \mathbf{S}$ & $\mathbf{S} / \mathbf{P}$ & O.C. & $\mathbf{Y} / \mathbf{P}$ \\
\hline 1X2 & $13.12 *$ & $1.15^{*}$ & -0.37 & -2.03 & $-9.87 * *$ & -4.51 & -0.19 & $-1.87 * * *$ & 63.44 & -1.46 & -3.70 \\
\hline $1 \mathrm{X} 3$ & 0.16 & -0.10 & -0.91 & -3.26 & -1.40 & -0.57 & $-0.43^{*}$ & -0.003 & 11.63 & -1.10 & -0.39 \\
\hline $1 \times 4$ & -2.04 & 0.49 & 1.76 & $6.79 *$ & $10.06 * *$ & 3.76 & 0.33 & 0.47 & $95.56 * *$ & 0.37 & 2.93 \\
\hline $1 X 5$ & 1.12 & 0.03 & 0.13 & 0.44 & 1.18 & 0.17 & 0.40 & 0.03 & -59.75 & 0.06 & 5.75 \\
\hline $1 \times 6$ & -2.36 & -0.13 & 0.44 & 0.03 & 5.22 & 4.21 & 0.16 & -0.67 & 10.54 & 2.21 & 1.95 \\
\hline $1 X 7$ & -10.26 & -0.62 & -0.08 & -5.56 & -2.08 & -1.14 & -0.34 & -0.48 & -29.91 & -0.39 & 3.16 \\
\hline $1 X 8$ & 9.64 & -0.27 & -1.23 & 2.63 & -1.95 & -0.83 & 0.004 & 0.48 & -67.61 & 0.28 & 0.24 \\
\hline $1 \times 9$ & 2.76 & 0.42 & 1.24 & 4.19 & 1.88 & -3.63 & 0.05 & 0.33 & -3.35 & 0.61 & -2.26 \\
\hline $1 \mathrm{X} 10$ & -7.20 & -0.08 & 1.47 & 1.56 & 4.67 & 3.59 & 0.01 & 0.26 & 15.87 & 2.52 & $6.70^{*}$ \\
\hline $2 \times 3$ & 11.40 & -0.10 & 1.21 & 2.11 & -0.41 & 0.18 & -0.15 & -0.50 & 18.40 & -1.25 & 2.24 \\
\hline $2 \times 4$ & 1.54 & $-0.92 *$ & -1.92 & -2.51 & -3.15 & 3.64 & -0.05 & $-0.89 *$ & -21.68 & -0.65 & -3.58 \\
\hline $2 \times 5$ & -3.64 & -0.17 & 0.25 & -0.20 & -2.50 & -2.02 & 0.38 & 0.48 & -14.32 & 0.07 & -0.62 \\
\hline $2 \times 6$ & -0.32 & -0.01 & -0.32 & 0.72 & 4.61 & 2.96 & -0.05 & 0.03 & -11.90 & 1.99 & -0.43 \\
\hline $2 \times 7$ & 5.32 & 0.38 & 2.03 & 1.47 & 5.84 & 0.94 & -0.26 & 0.30 & 43.12 & 0.14 & 5.82 \\
\hline $2 \times 8$ & 8.21 & -0.08 & 1.15 & 6.19 & 5.38 & $5.19 *$ & -0.04 & -0.31 & -6.71 & -0.83 & 3.37 \\
\hline $2 \times 9$ & 0.33 & 0.15 & 1.83 & -3.78 & -4.13 & -1.28 & -0.09 & 0.64 & 54.01 & -0.36 & 2.20 \\
\hline 2X10 & 4.71 & 0.52 & -0.28 & 2.72 & 1.99 & 0.14 & 0.12 & 0.14 & -10.90 & 2.70 & 0.76 \\
\hline $3 X 4$ & -5.85 & -0.16 & -0.79 & $-6.73 *$ & $-8.69 *$ & $-6.62 * *$ & 0.004 & 0.15 & -68.22 & 0.65 & -1.86 \\
\hline $3 \times 5$ & -2.69 & 0.38 & -0.42 & 1.91 & $7.04 *$ & $8.52 * * *$ & -0.05 & -0.20 & -10.86 & -0.77 & -4.24 \\
\hline $3 \times 6$ & $16.63 * *$ & 0.42 & $5.68 * * *$ & $8.50 *$ & 1.74 & 2.84 & $-0.65 * *$ & $-2.23 * * *$ & 41.97 & 1.85 & -5.88 \\
\hline $3 \times 7$ & -0.41 & 0.13 & 1.10 & 2.58 & -1.36 & -2.05 & $0.46^{*}$ & 0.10 & -3.62 & 1.86 & 0.46 \\
\hline $3 \times 8$ & -1.18 & 0.08 & -0.32 & 3.97 & 6.51 & 1.93 & 0.19 & $1.00 *$ & 15.49 & -1.27 & 3.41 \\
\hline $3 \times 9$ & -6.06 & 0.24 & 1.23 & -0.67 & -0.66 & 1.66 & 0.35 & 0.48 & 15.14 & -1.14 & 0.51 \\
\hline $3 \times 10$ & 2.65 & -0.20 & -1.34 & 2.50 & 3.79 & 2.55 & 0.20 & 0.19 & 0.63 & 2.16 & 4.90 \\
\hline $4 X 5$ & -3.55 & 0.17 & -0.48 & -0.04 & -3.04 & -1.42 & 0.09 & 0.002 & 29.34 & 0.77 & -0.03 \\
\hline $4 \times 6$ & -11.24 & -0.47 & -1.31 & $-8.45^{*}$ & -6.66 & $-7.24 * *$ & 0.05 & 0.43 & -64.31 & 3.86 & -3.53 \\
\hline $4 X 7$ & 9.73 & 0.65 & 0.97 & $9.63 * *$ & $8.24 *$ & $5.88 *$ & 0.21 & -0.19 & 17.84 & 2.30 & 1.68 \\
\hline $4 X 8$ & 4.37 & 0.46 & $-2.31 *$ & $-7.65^{*}$ & $-9.56 * *$ & -4.01 & -0.26 & -0.31 & -25.05 & 0.67 & -2.94 \\
\hline $4 \times 9$ & 7.08 & 0.29 & 0.76 & 1.71 & 4.60 & -0.35 & -0.09 & 0.03 & -18.73 & 1.17 & -0.64 \\
\hline $4 \times 10$ & 8.79 & -0.41 & 0.06 & 1.55 & -0.95 & 1.61 & -0.13 & -0.26 & -5.51 & $-13.64 * * *$ & 0.79 \\
\hline $5 \times 6$ & $-19.08 * *$ & -0.26 & -0.88 & -6.14 & -1.81 & 0.51 & 0.02 & 0.55 & -23.02 & 2.14 & -0.94 \\
\hline $5 \times 7$ & 0.69 & 0.07 & 0.67 & 0.03 & 0.59 & 0.98 & 0.08 & -0.23 & 2.37 & -0.05 & -2.96 \\
\hline $5 \times 8$ & 9.46 & 0.20 & 1.12 & $8.00 *$ & $13.29 * * *$ & 4.26 & 0.43 & 0.02 & 51.04 & -0.61 & 5.55 \\
\hline $5 \times 9$ & 3.76 & 0.37 & -0.67 & 5.56 & 4.12 & 1.73 & 0.32 & 0.36 & -31.57 & -0.45 & 4.12 \\
\hline
\end{tabular}




\begin{tabular}{|c|c|c|c|c|c|c|c|c|c|c|c|}
\hline $5 \times 10$ & -12.38 & -0.34 & 0.50 & -5.47 & -2.09 & -4.18 & $0.44 *$ & 0.29 & 8.71 & 2.18 & -1.69 \\
\hline $6 \times 7$ & 8.88 & 0.03 & -0.42 & -0.94 & $-8.34 *$ & -3.27 & -0.12 & 0.10 & -13.65 & $-13.03 * * *$ & -1.57 \\
\hline $6 \times 8$ & -1.89 & -0.03 & 0.63 & 2.59 & 0.20 & 3.78 & 0.12 & 0.39 & 22.00 & 1.01 & 0.98 \\
\hline $6 \times 9$ & 6.69 & 0.27 & -0.17 & -0.73 & -6.97 & -2.96 & -0.06 & -0.13 & -20.15 & 2.31 & 1.55 \\
\hline $6 \times 10$ & 4.48 & -0.17 & $-2.37 *$ & 2.20 & 0.48 & -0.60 & 0.26 & 0.13 & -13.50 & $-12.93 * * *$ & -2.96 \\
\hline $7 \times 8$ & $-14.03 *$ & -0.58 & -0.39 & -3.66 & -2.99 & -2.95 & 0.05 & 0.29 & 10.23 & 0.85 & -4.18 \\
\hline $7 \times 9$ & 9.19 & 0.25 & $-5.35 * * *$ & -5.98 & $-7.74 *$ & -2.65 & $-0.61 * *$ & -0.85 & -42.40 & 0.35 & -5.44 \\
\hline 7X10 & 0.09 & 0.48 & 0.82 & -0.14 & 0.05 & -0.23 & 0.28 & 0.46 & -30.85 & 3.51 & $-7.05 *$ \\
\hline $8 \times 9$ & 0.42 & -0.34 & 0.50 & 1.22 & 1.93 & 2.20 & 0.38 & -0.52 & -35.90 & -0.82 & -0.33 \\
\hline 8X10 & 11.17 & 0.49 & -0.27 & 5.92 & -0.08 & -1.18 & $0.54 *$ & 0.68 & 21.99 & 2.25 & -2.04 \\
\hline 9X10 & -2.41 & -0.21 & 0.27 & 4.28 & 3.74 & -0.58 & 0.09 & -0.24 & -2.56 & 2.45 & 0.16 \\
\hline
\end{tabular}

Table.4 Estimates of heterosis for 11 character in 10 X 10 half diallel set of Brassica juncea (L.) Czern and Coss

\begin{tabular}{|c|c|c|c|c|c|c|c|c|c|c|c|c|}
\hline Crosses & $\begin{array}{c}\text { Heterosis } \\
(\%)\end{array}$ & PH & PB & SB & FZL & MSL & SOMS & SL & $\mathbf{S} / \mathbf{S}$ & $\mathbf{S} / \mathbf{P}$ & O.C. & $\mathbf{Y} / \mathbf{P}$ \\
\hline \multirow{2}{*}{$\begin{array}{c}\text { BPR 543-2 X } \\
\text { Urvashi }\end{array}$} & BP & $-14.13 * *$ & $25.00 *$ & 3.16 & -1.88 & $-14.17 *$ & -7.16 & -6.90 & $-16.84 * *$ & 26.62 & $-3.77 * *$ & 7.97 \\
\hline & MP & -7.68 & $29.63 * *$ & 11.64 & 0.38 & -11.02 & -6.48 & -4.70 & $-16.67 * *$ & $33.36^{*}$ & -2.11 & 8.51 \\
\hline \multirow{2}{*}{$\begin{array}{c}\text { BPR 543-2 X } \\
\text { BPR 549-9 }\end{array}$} & $\mathrm{BP}$ & -5.78 & 3.57 & 0.00 & 0.00 & -2.03 & 2.15 & -8.77 & -4.72 & 6.59 & -2.14 & -2.79 \\
\hline & MP & -0.76 & 5.45 & 11.27 & 0.76 & 2.77 & 3.94 & -7.33 & -3.90 & 8.24 & -0.74 & 14.83 \\
\hline \multirow{2}{*}{$\begin{array}{c}\text { BPR 543-2 X } \\
\text { DRMR 1165-40 }\end{array}$} & $\mathrm{BP}$ & -7.22 & 11.63 & 10.65 & 4.42 & 4.50 & -3.32 & 4.73 & -2.03 & 21.55 & 0.35 & 10.17 \\
\hline & MP & -3.67 & 12.94 & 14.37 & 7.21 & $12.10 *$ & 5.72 & 6.54 & -0.25 & 26.86 & 1.60 & 24.78 \\
\hline \multirow{2}{*}{$\begin{array}{l}\text { BPR 543-2 X } \\
\text { UP-II-73 }\end{array}$} & $\mathrm{BP}$ & -6.42 & 5.88 & 6.96 & 0.38 & 6.01 & -1.93 & 0.89 & -5.04 & -20.26 & $3.37 *$ & 57.20 \\
\hline & MP & -6.27 & 6.51 & 7.64 & 3.09 & 10.21 & 6.18 & $18.87 * *$ & 0.00 & -19.79 & $3.42 *$ & $61.95 *$ \\
\hline \multirow{2}{*}{$\begin{array}{c}\text { BPR 543-2 X } \\
\text { EC-511664 }\end{array}$} & $\mathrm{BP}$ & -8.57 & -5.32 & 6.21 & -0.73 & -3.45 & 3.15 & -0.21 & $-8.62 *$ & -8.22 & -1.00 & -12.57 \\
\hline & MP & -4.75 & 0.00 & 12.24 & 0.74 & 5.22 & 9.47 & 1.56 & $-8.44 *$ & 0.46 & 0.16 & 12.25 \\
\hline \multirow{2}{*}{$\begin{array}{c}\text { BPR 543-2 X } \\
\text { NRCDR 02 }\end{array}$} & BP & $-11.08 *$ & -3.57 & -1.27 & -7.25 & -9.22 & -10.54 & -8.41 & -6.59 & -10.71 & -0.34 & 0.54 \\
\hline & MP & -6.89 & -3.57 & 3.65 & -5.54 & -2.62 & -4.13 & -6.81 & -5.98 & -10.26 & 0.02 & 20.48 \\
\hline \multirow{2}{*}{$\begin{array}{l}\text { BPR 543-2 X } \\
\text { NRCHB } 101\end{array}$} & $\mathrm{BP}$ & -4.17 & -2.38 & -10.13 & 0.53 & 1.35 & 3.25 & -3.27 & -2.77 & -20.34 & -0.71 & 20.61 \\
\hline & MP & 4.55 & -1.20 & -8.68 & 10.72 & 4.39 & 3.41 & 6.76 & 3.46 & -19.67 & 1.18 & 27.58 \\
\hline \multirow{2}{*}{$\begin{array}{l}\text { BPR 543-2 X } \\
\text { DRMR IJ-31 }\end{array}$} & $\mathrm{BP}$ & -2.89 & 16.67 & 13.94 & 6.64 & -1.61 & $-15.87 *$ & 0.23 & -0.90 & -14.97 & 0.93 & -11.20 \\
\hline & MP & 0.50 & 18.07 & 16.41 & 7.64 & 3.81 & -10.01 & 2.49 & -0.04 & -4.29 & 1.50 & 6.30 \\
\hline \multirow{2}{*}{$\begin{array}{c}\text { BPR 543-2 X } \\
\text { ROHINI }\end{array}$} & $\mathrm{BP}$ & $-11.24 *$ & 1.19 & 16.46 & -1.28 & 8.52 & 8.61 & -2.76 & -1.71 & 3.58 & 1.22 & 51.84 \\
\hline & MP & -6.27 & 3.03 & 17.20 & 8.07 & $13.35^{*}$ & 9.25 & 8.75 & 1.98 & 6.77 & 2.03 & $58.69 *$ \\
\hline \multirow{2}{*}{$\begin{array}{c}\text { URVASHI X } \\
\text { BPR-549-9 }\end{array}$} & $\mathrm{BP}$ & 7.86 & 3.70 & 35.82 & 5.34 & -0.41 & 6.73 & -5.35 & $-8.42 *$ & 13.17 & -2.47 & -0.14 \\
\hline & MP & $10.22 *$ & 5.66 & $40.00 *$ & 6.98 & 0.82 & 7.81 & -4.61 & $-7.81 *$ & 17.44 & -2.19 & 17.47 \\
\hline \multirow{2}{*}{$\begin{array}{l}\text { URVASHI X } \\
\text { DRMR 1165-40 }\end{array}$} & $\mathrm{BP}$ & -0.87 & -16.28 & -26.63 & -7.70 & -10.47 & -0.98 & -2.32 & $-10.95 * *$ & -11.26 & $-4.51 * *$ & -29.12 \\
\hline & MP & 2.79 & -12.20 & -18.15 & -3.11 & -7.23 & 7.55 & -1.70 & $-9.51 * *$ & -2.68 & $-4.05 * *$ & -20.07 \\
\hline URVASHI X & $\mathrm{BP}$ & $-11.11 *$ & -1.18 & 4.49 & 1.97 & -5.67 & -5.12 & 1.22 & -2.94 & -3.87 & 0.55 & 9.66 \\
\hline
\end{tabular}




\begin{tabular}{|c|c|c|c|c|c|c|c|c|c|c|c|c|}
\hline UP-II-73 & MP & -4.58 & 3.07 & 12.41 & 2.37 & 1.52 & 3.43 & $16.93 * *$ & 2.02 & 0.68 & $2.33 *$ & 12.43 \\
\hline \multirow{2}{*}{$\begin{array}{c}\text { URVASHI X } \\
\text { EC-511664 }\end{array}$} & $\mathrm{BP}$ & -2.44 & -6.38 & -4.52 & -2.19 & -3.75 & 3.41 & -4.19 & -4.98 & -13.22 & -1.93 & -27.27 \\
\hline & MP & 0.81 & 2.33 & 8.68 & 1.52 & 1.38 & 8.99 & -3.62 & -4.98 & -0.46 & -1.39 & -6.96 \\
\hline \multirow{2}{*}{$\begin{array}{l}\text { URVASHI X } \\
\text { NRCDR } 02\end{array}$} & $\mathrm{BP}$ & 3.00 & 10.71 & 25.87 & -1.81 & 0.39 & -3.86 & -7.69 & -2.45 & 14.35 & $-3.03 *$ & 3.23 \\
\hline & MP & 5.89 & 14.81 & 29.96 & 2.26 & 4.02 & 2.33 & -7.12 & -2.01 & 19.86 & -1.72 & 23.21 \\
\hline \multirow{2}{*}{$\begin{array}{l}\text { URVASHI X } \\
\text { NRCHB } 101\end{array}$} & $\mathrm{BP}$ & 7.46 & 0.00 & 11.11 & 7.09 & 3.75 & $18.27 *$ & -3.31 & $-8.25^{*}$ & 1.47 & -1.34 & 26.70 \\
\hline & MP & 9.19 & 2.50 & 18.47 & $15.50 * *$ & 10.67 & $18.97 * *$ & 4.45 & -2.56 & 6.01 & -1.16 & 33.40 \\
\hline \multirow{2}{*}{$\begin{array}{l}\text { URVASHI X } \\
\text { DRMR IJ-31 }\end{array}$} & $\mathrm{BP}$ & 0.00 & 10.98 & 14.55 & -4.43 & -8.43 & -8.65 & -1.72 & 0.41 & -0.21 & -0.20 & -1.21 \\
\hline & MP & 4.03 & 13.75 & 26.42 & -1.33 & -6.75 & -2.95 & -1.62 & 1.07 & 17.52 & 0.96 & 17.78 \\
\hline \multirow{2}{*}{$\begin{array}{l}\text { URVASHI X } \\
\text { ROHINI }\end{array}$} & $\mathrm{BP}$ & 2.69 & 12.35 & -3.85 & 2.36 & -2.08 & 2.49 & -0.19 & -3.10 & 2.29 & 0.48 & 8.09 \\
\hline & MP & 4.67 & 14.47 & 3.45 & 9.70 & 5.86 & 3.85 & 9.30 & 0.34 & 4.59 & 1.40 & 12.43 \\
\hline \multirow{2}{*}{$\begin{array}{c}\text { BPR-549-9 X } \\
\text { DRMR 1165-40 }\end{array}$} & $\mathrm{BP}$ & -2.60 & -2.33 & -14.79 & -9.12 & $-13.18^{*}$ & $-17.59 * *$ & 0.19 & -2.57 & -28.09 & -0.71 & -20.92 \\
\hline & MP & -1.14 & 0.60 & -2.37 & -6.01 & $-11.11^{*}$ & -11.32 & 0.34 & -1.63 & -23.84 & -0.53 & -16.98 \\
\hline \multirow{2}{*}{$\begin{array}{l}\text { BPR-549-9 X } \\
\text { UP-II-73 }\end{array}$} & $\mathrm{BP}$ & -8.70 & 9.41 & 0.00 & 4.58 & 7.56 & $18.34 *$ & -5.97 & -5.13 & -6.58 & -0.76 & -26.64 \\
\hline & MP & -3.98 & 12.05 & 10.64 & 6.61 & $17.08 * *$ & $30.18 * *$ & 9.35 & -0.91 & -5.69 & 0.71 & -15.50 \\
\hline \multirow{2}{*}{$\begin{array}{c}\text { BPR-549-9 X } \\
\text { EC-511664 }\end{array}$} & $\mathrm{BP}$ & 8.55 & 1.06 & $48.02 * *$ & 9.49 & -3.37 & 5.64 & $-13.63^{*}$ & $-18.06^{* *}$ & -2.35 & -1.53 & $-39.39 *$ \\
\hline & MP & $9.80 *$ & 8.57 & $72.94 * *$ & $11.94 *$ & 0.58 & 10.27 & $-13.47 * *$ & $-17.52 * *$ & 8.39 & -1.28 & -32.84 \\
\hline \multirow{2}{*}{$\begin{array}{c}\text { BPR-549-9 } \\
\text { X NRCDR 02 }\end{array}$} & BP & 2.12 & 7.14 & 18.18 & 2.54 & -4.26 & -7.20 & 6.25 & -2.06 & -4.27 & 1.77 & -10.90 \\
\hline & MP & 2.75 & 9.09 & 25.65 & 5.20 & -1.98 & -2.17 & 6.42 & -1.86 & -3.27 & $2.86^{*}$ & -9.32 \\
\hline \multirow{2}{*}{$\begin{array}{l}\text { BPR-549-9 X } \\
\text { NRCHB 101 }\end{array}$} & BP & 0.00 & 3.66 & -1.31 & 4.58 & 6.50 & 11.60 & 1.01 & 1.41 & 4.72 & -2.30 & 6.56 \\
\hline & MP & 3.80 & 4.29 & 8.24 & $14.41 *$ & $14.91 *$ & 13.39 & 9.90 & 7.03 & 5.47 & -1.84 & 19.84 \\
\hline \multirow{2}{*}{$\begin{array}{l}\text { BPR-549-9 X } \\
\text { DRMR IJ-31 }\end{array}$} & BP & -1.20 & 13.41 & 10.91 & 2.21 & -0.40 & -0.52 & 6.65 & 1.49 & -12.61 & -1.23 & -0.54 \\
\hline & MP & 0.61 & 14.11 & 25.77 & 3.94 & 0.20 & 4.68 & 7.37 & 1.49 & -0.31 & -0.38 & 1.10 \\
\hline \multirow{2}{*}{$\begin{array}{l}\text { BPR-549-9 X } \\
\text { ROHINI }\end{array}$} & $\mathrm{BP}$ & 3.21 & 0.00 & -12.18 & 2.29 & 1.63 & 8.31 & 1.58 & -0.75 & -0.95 & -0.15 & 7.11 \\
\hline & MP & 3.49 & 0.00 & -2.84 & $11.20 *$ & 11.11 & 10.85 & $12.03 *$ & 2.13 & 0.57 & 0.48 & 21.81 \\
\hline \multirow{2}{*}{$\begin{array}{l}\text { DRMR 1165-40 X } \\
\text { UP-II-73 }\end{array}$} & $\mathrm{BP}$ & $-9.50 *$ & 4.65 & -14.79 & -5.56 & -11.40 & $-15.74 *$ & -2.37 & -2.19 & -4.10 & -0.16 & -12.02 \\
\hline & MP & -6.18 & 5.26 & -11.38 & -0.49 & -1.47 & -1.01 & $13.39 *$ & 1.22 & 0.64 & 1.13 & -2.97 \\
\hline \multirow{2}{*}{$\begin{array}{c}\text { DRMR 1165-40 X } \\
\text { EC-511664 }\end{array}$} & $\mathrm{BP}$ & -7.11 & -12.77 & -17.51 & $-12.33^{*}$ & $-14.98^{*}$ & $-18.57 * *$ & 0.14 & -1.96 & $-30.18^{*}$ & 0.09 & $-40.63 *$ \\
\hline & MP & -6.78 & -8.89 & -15.61 & $-11.29 *$ & $-13.52 *$ & $-15.94 * *$ & 0.18 & -0.37 & $-26.61 *$ & 0.15 & -31.30 \\
\hline \multirow{2}{*}{$\begin{array}{l}\text { DRMR 1165-40 X } \\
\text { NRCDR 02 }\end{array}$} & BP & 5.20 & 13.95 & -7.69 & 7.27 & 4.65 & 4.43 & 3.36 & -4.04 & -7.25 & -0.34 & -16.96 \\
\hline & MP & 6.12 & 15.29 & 0.00 & 8.16 & 4.65 & 6.73 & 3.37 & -2.92 & -2.73 & 0.53 & -11.35 \\
\hline \multirow{2}{*}{$\begin{array}{c}\text { DRMR 1165-40 X } \\
\text { NRCHB } 101\end{array}$} & BP & -5.03 & 5.81 & $-34.91 *$ & $-15.89 * *$ & $-19.38 * *$ & $-14.15^{*}$ & -5.72 & -4.64 & -17.56 & -1.22 & -24.19 \\
\hline & MP & 0.00 & 8.33 & $-31.68 *$ & -5.14 & -11.11 & -6.25 & 2.43 & -0.26 & -13.27 & -0.57 & -18.48 \\
\hline \multirow{2}{*}{$\begin{array}{l}\text { DRMR 1165-40 X } \\
\text { DRMR IJ-31 }\end{array}$} & BP & 5.16 & 9.30 & -2.37 & 0.14 & 0.00 & -7.87 & 0.07 & -1.49 & -21.10 & 1.12 & -16.19 \\
\hline & MP & 5.53 & 11.90 & -1.20 & 1.89 & 1.78 & -5.67 & 0.59 & -0.54 & -14.59 & 1.80 & -10.65 \\
\hline \multirow{2}{*}{$\begin{array}{c}\text { DRMR 1165-40 X } \\
\text { ROHINI }\end{array}$} & BP & 2.95 & -9.30 & -13.02 & -6.63 & -10.85 & -7.75 & -3.00 & -1.86 & -14.04 & -0.04 & -13.25 \\
\hline & MP & 4.76 & -6.59 & -9.54 & 4.67 & -0.43 & 1.42 & 6.82 & 0.04 & -7.64 & 0.40 & -5.62 \\
\hline \multirow{2}{*}{$\begin{array}{l}\text { UP-II-73 X } \\
\text { EC-511664 }\end{array}$} & BP & $-18.20 * *$ & -8.51 & -10.17 & -10.22 & $-11.99 *$ & -7.22 & -5.59 & -1.63 & -22.90 & -0.43 & -28.51 \\
\hline & MP & $-14.91 * *$ & -3.91 & -4.50 & -6.46 & -0.63 & 6.08 & 9.62 & 3.39 & -15.15 & 0.79 & -10.22 \\
\hline \multirow{2}{*}{$\begin{array}{l}\text { UP-II-73 X } \\
\text { NRCDR } 02\end{array}$} & BP & -8.01 & 6.18 & 1.28 & -3.89 & -6.78 & -9.45 & -4.25 & -4.66 & -6.86 & -0.71 & -31.63 \\
\hline & MP & -3.83 & 6.80 & 5.69 & 0.47 & 3.66 & 4.45 & 11.19 & -0.22 & -6.78 & -0.30 & -20.06 \\
\hline
\end{tabular}




\begin{tabular}{|c|c|c|c|c|c|c|c|c|c|c|c|c|}
\hline \multirow{2}{*}{$\begin{array}{l}\text { UP-II-73 X } \\
\text { NRCHB } 101\end{array}$} & BP & -5.80 & 3.53 & 7.69 & 9.52 & $28.57 * *$ & 11.09 & $20.48 * *$ & 4.07 & 10.99 & -0.75 & 39.25 \\
\hline & MP & 2.63 & 5.39 & 8.74 & $17.70 * *$ & $29.81 * *$ & $20.45 * *$ & $29.63 * *$ & 5.22 & 11.27 & 1.19 & 43.09 \\
\hline \multirow{2}{*}{$\begin{array}{l}\text { UP-II-73 X } \\
\text { DRMR IJ-31 }\end{array}$} & BP & -7.57 & 12.94 & -9.09 & 5.39 & 0.40 & -8.52 & 3.25 & 0.17 & $-27.45 *$ & 2.06 & 7.15 \\
\hline & MP & -4.49 & 14.97 & -6.54 & 9.22 & 9.89 & 5.35 & $19.39 * *$ & 4.63 & -17.92 & $2.69 *$ & 25.14 \\
\hline \multirow{2}{*}{$\begin{array}{c}\text { UP-II-73 X } \\
\text { ROHINI }\end{array}$} & BP & $-15.30 * *$ & -5.88 & 2.56 & -7.14 & 6.80 & -11.11 & $24.58 * *$ & 5.26 & -4.76 & 1.15 & -4.60 \\
\hline & MP & $-10.70 *$ & -3.61 & 2.56 & -0.85 & 7.32 & -4.28 & $32.19 * *$ & 6.90 & -2.39 & 2.02 & -3.17 \\
\hline \multirow{2}{*}{$\begin{array}{c}\text { EC-511664 X } \\
\text { NRCDR } 02\end{array}$} & $\mathrm{BP}$ & 4.19 & -3.19 & -11.86 & -2.68 & $-16.48 * *$ & -9.38 & -4.45 & -2.78 & -20.32 & 0.95 & -31.20 \\
\hline & MP & 4.74 & 2.25 & -2.50 & -2.33 & $-15.05^{* *}$ & -8.44 & -4.42 & -2.34 & -12.38 & 1.76 & -24.97 \\
\hline \multirow{2}{*}{$\begin{array}{l}\text { EC-511664 X } \\
\text { NRCHB } 101\end{array}$} & $\mathrm{BP}$ & -4.36 & -8.51 & -1.13 & -2.92 & -10.11 & 6.04 & -0.90 & -3.10 & -9.24 & $-3.97 * *$ & -21.10 \\
\hline & MP & 0.37 & -2.27 & 6.06 & 8.35 & 0.63 & 12.38 & 7.63 & 2.91 & 0.10 & $-3.27 * *$ & -2.97 \\
\hline \multirow{2}{*}{$\begin{array}{l}\text { EC-511664 X } \\
\text { DRMR IJ-31 }\end{array}$} & $\mathrm{BP}$ & 3.68 & 2.13 & -2.82 & -0.36 & $-15.36^{*}$ & -9.29 & -1.37 & -3.43 & -17.56 & 0.33 & -12.46 \\
\hline & MP & 4.40 & 9.09 & 0.58 & 0.18 & $-12.40 *$ & -8.52 & -0.89 & -2.80 & -14.97 & 0.94 & -4.41 \\
\hline \multirow{2}{*}{$\begin{array}{r}\text { EC-511664 X } \\
\text { ROHINI }\end{array}$} & $\mathrm{BP}$ & -4.58 & -10.64 & -25.85 & -3.92 & -11.24 & -6.82 & 1.98 & -2.29 & -20.34 & -1.63 & $-38.95 *$ \\
\hline & MP & -3.23 & -4.00 & -21.17 & 6.58 & 0.64 & -0.56 & $12.27 *$ & 1.18 & -10.38 & -1.27 & -24.18 \\
\hline \multirow{2}{*}{$\begin{array}{l}\text { NRCDR 02 X } \\
\text { NRCHB } 101\end{array}$} & $\mathrm{BP}$ & -8.35 & -8.33 & -11.44 & -10.14 & -11.34 & -10.35 & -1.98 & -1.92 & -1.96 & -1.05 & -35.40 \\
\hline & MP & -4.30 & -7.23 & -8.45 & 0.61 & -2.24 & -4.06 & 6.49 & 3.73 & -1.63 & 0.47 & -26.21 \\
\hline \multirow{2}{*}{$\begin{array}{l}\text { NRCDR } 02 \text { X } \\
\text { DRMR IJ-31 }\end{array}$} & $\mathrm{BP}$ & 6.37 & 13.10 & $-57.58 * *$ & -6.52 & $-13.95 *$ & -10.28 & -10.55 & -6.10 & $-29.96^{*}$ & 0.11 & -32.44 \\
\hline & MP & 7.67 & 14.46 & $-54.55 * *$ & -5.67 & $-12.43 *$ & -10.11 & $-10.09 *$ & -5.91 & -20.81 & 0.31 & -32.35 \\
\hline \multirow{2}{*}{$\begin{array}{l}\text { NRCDR 02 X } \\
\text { ROHINI }\end{array}$} & BP & 0.18 & 10.71 & -0.64 & -6.88 & -9.30 & -9.25 & 2.58 & 1.57 & -17.29 & 0.48 & $-52.49 *$ \\
\hline & MP & 1.07 & 12.73 & 3.68 & 3.63 & 1.30 & -2.22 & $12.96 *$ & 4.72 & -15.16 & 0.91 & $-45.14 *$ \\
\hline \multirow{2}{*}{$\begin{array}{l}\text { NRCHB } 101 \text { X } \\
\text { DRMR IJ-31 }\end{array}$} & BP & -1.03 & 0.00 & -2.42 & -1.70 & -2.65 & -0.90 & 7.12 & -5.79 & $-26.66 *$ & -0.90 & -9.72 \\
\hline & MP & 4.55 & 0.00 & 1.26 & 9.18 & 5.62 & 5.86 & $15.83 * *$ & -0.57 & -16.84 & 0.43 & 3.00 \\
\hline \multirow{2}{*}{$\begin{array}{c}\text { NRCHB } 101 \mathrm{X} \\
\text { ROHINI }\end{array}$} & $\mathrm{BP}$ & 5.03 & 8.54 & -8.97 & $19.09 * *$ & 7.14 & 1.92 & $25.82 * *$ & 7.28 & 2.38 & -0.42 & -7.35 \\
\hline & MP & 8.74 & 9.20 & -8.09 & $19.91 * *$ & 8.70 & 2.68 & $27.71 * *$ & $10.13 * *$ & 4.68 & 0.68 & -6.17 \\
\hline \multirow{2}{*}{$\begin{array}{c}\text { DRMR IJ-31 X } \\
\text { ROHINI }\end{array}$} & $\mathrm{BP}$ & -1.38 & 2.44 & -3.64 & 1.11 & -2.01 & -10.45 & 2.39 & -1.57 & -20.30 & 2.06 & -13.23 \\
\hline & MP & 0.70 & 3.07 & -0.93 & $11.61^{*}$ & 7.73 & -3.68 & $12.23 *$ & 1.28 & -7.88 & $2.30 *$ & 0.08 \\
\hline \multirow[t]{2}{*}{ S.E. d } & $\mathrm{BP}$ & 9.45 & 0.66 & 1.76 & 5.12 & 5.34 & 3.58 & 0.33 & 0.65 & 52.84 & 0.55 & 5.09 \\
\hline & MP & 8.18 & 0.57 & 1.53 & 4.43 & 4.63 & 3.10 & 0.28 & 0.56 & 45.76 & 0.47 & 4.41 \\
\hline \multirow[t]{2}{*}{ CD 5\% } & BP & 18.72 & 1.30 & 3.49 & 10.14 & 10.59 & 7.09 & 0.65 & 1.29 & 104.73 & 1.08 & 10.26 \\
\hline & MP & 16.21 & 1.13 & 3.02 & 8.78 & 9.17 & 6.14 & 0.56 & 1.12 & 90.70 & 0.94 & 8.89 \\
\hline \multirow[t]{2}{*}{ CD 1\% } & BP & 24.77 & 1.72 & 4.62 & 13.42 & 14.01 & 9.38 & 0.86 & 1.71 & 138.54 & 1.43 & 13.35 \\
\hline & MP & 21.45 & 1.49 & 4.00 & 11.62 & 12.13 & 8.13 & 0.75 & 1.48 & 119.98 & 1.24 & 11.56 \\
\hline
\end{tabular}




\section{Acknowledgements}

Author sincerely acknowledges the grant received under Incentivizing Research in Agriculture project under which this study conducted.

\section{References}

Allard, R. W. 1960. Principles of Plant Breeding. New York: John willey and Sons. New York.

Banga, S. S. 2012. Germplasm Enhancement in Indian Mustard: Some Exiting New Developments. In: "Souvenir of XIX Annual AICRP Group Meet on RapeseedMustard", Birsa Agricultural University, Ranchi, India, PP. 29-34.

Briggle, L.W. 1963. Heterosis in Wheat - A review. Crop Sci., 3(3): 407-412.

Fonseca, S. and Patterson, F.L. 1968. Hybrid vigour in a seven parents diallel crosses in common winter wheat (Triticum aestivum L.). Crop Sci., 8: 85-88.

Griffing, B. 1956. A generalized treatment of the use of diallel crosses in quantitative inheritance. Heredity, 10: 31-50.

Gupta Priti, Chaudhary and Sandeep Kumar Lal, 2011. Heterosis and combining ability analysis for yield and its components in Indian mustard (Brassica juncea L. Czern \& Coss ). Academic J. Plant Sci., 4(2): 4552.

Khulbe, R.K., D.P. Part and R.S. Rawat, 1998. Heterosis for yield and its components in Indian mustard. J. Oilseed Res., 15: 227230.

Mahmood, T., M. Ali, M. Anwar and S. Iqbal, 2003. Heterosis for some quantitative characters in Brassica juncea (L.). Asian J. Plant Sci., 2(1): 71-73.

Mahto, J.L. and Z.A. Haider 2001. Assessing suitable combiners in [Brassica juncea (L.) Czern \& Coss] for high altitude acidic soils.Cruciferae Newslr., 23: 47-48.

Mahto, J.L., and Z.A. Haider, 2004. Heterosis in Indian mustard (Brassica juncea L. Czern
\& Coss ). J. Tropical Agric., 42(1-2): 3941.

Meena, H.S., Kumar, A, Ram, B., Singh, V.V., Singh, B. K., Meena, P.D. and Singh, D. 2015. Combining ability and heterosis for seed yield and its components in Indian mustard (Brassica juncea). Journal of Agricultural Science and Technology 17: 1861-1871.

Meena, H.S., Ram, B., Kumar, A., Singh, B. K., Meena, P.D., Singh, V.V. and Singh, D. 2014. Heterobeltiosis and standard heterosis for seed yield and important traits in Brassica juncea. Journal of Oilseed Brassica, 5(2): 134-140.

Panse, V.G. and P.V. Sukhatme, 1967. Statistical Methods for Agricultural Workers.2nd edn. ICAR. New Delhi.

Patel, A.M., D.B. Prajapati, and D.G. Patel, 2012. Heterosis and combining ability studies in Indian mustard (Brassica juncea L.). Ind. J. Sci. Res. and Tech., 1(1): 38-40.

Sheikh, I.A. and J.N. Singh, 1998. Combining ability analysis for seed yield and oil content in [Brassica juncea (L.)Czern\&Coss].Indian J. Genet., 58 (4): 507-511.

Singh M, A.H. Basharat, Lokendra Singh, B.Singh and R.K. Dixit (2008). Combining ability analysis for oil contents, seed yield and it's contributing characters in Indian mustard (Brassica juncea (L.) Czern and Coss).Journal of Progressive Agriculture, 3(2): 147-150.

Singh, K.H., M.C. Gupta, K.K. Shrivastava, and P.R. Kumar, 2003a. Combining ability and heterosis in Indian mustard. J. Oilseeds Res., 20(1): 35-39.

Teklewold, A. and H.C. Becker, 2005. Heterosis and combining ability in a diallel cross of Ethiopian mustard inbred lines. Crop Sci. 45(6): 2629-2635.

Verma, O.P., G.D. Khushwala and H.P. Singh 2000. Heterosis in relation to genetic diversity in Indian mustard. Cruciferae Newsletter, 22: 93-94. 


\section{How to cite this article:}

Singh. V. V., Balbeer, H. S. Meena, Swarnim Kulshrestha, Monika Dubey, Neeraj Gurjar, Pankaj Garg, M. L. Meena and Rai. P. K. 2020. Heterosis and Combining Ability Analysis for Yield and Yield Attributes in Indian Mustard (Brassica juncea L.). Int.J.Curr.Microbiol.App.Sci. 9(03): 1622-1632. doi: https://doi.org/10.20546/ijcmas.2020.903.190 